\title{
Robotic Assistance for Ultrasound Guided Prostate Brachytherapy
}

\author{
Gabor Fichtinger ${ }^{1}$, Jonathan Fiene ${ }^{1}$, Christopher W. Kennedy ${ }^{1}$, Gernot Kronreif ${ }^{2}$, \\ Iulian Iordachita ${ }^{1}$, Danny Y. Song ${ }^{1}$, E. Clif Burdette ${ }^{3}$, and Peter Kazanzides ${ }^{1}$ \\ ${ }^{1}$ The Johns Hopkins University, Baltimore, Maryland, USA \\ ${ }^{2}$ PROFACTOR Research and Solutions GmbH, Seibersdorf, Austria \\ ${ }^{3}$ Acoustic Medsystems, Inc., Urbana-Champaign, IL, USA \\ gabor@cs.jhu.edu
}

\begin{abstract}
We present a robotically assisted prostate brachytherapy system and test results in training phantoms. The system consists of a transrectal ultrasound (TRUS) and a spatially co-registered robot integrated with an FDA-approved commercial treatment planning system. The salient feature of the system is a small parallel robot affixed to the mounting posts of the template. The robot replaces the template interchangeably and uses the same coordinate system. Established clinical hardware, workflow and calibration are left intact. In these experiments, we recorded the first insertion attempt without adjustment. All clinically relevant locations were reached. Non-parallel needle trajectories were achieved. The pre-insertion transverse and rotational errors (measured with Polaris optical tracker relative to the template's coordinate frame) were $0.25 \mathrm{~mm}$ $(\mathrm{STD}=0.17 \mathrm{~mm})$ and $0.75^{\circ}\left(\mathrm{STD}=0.37^{\circ}\right)$. The needle tip placement errors measured in TRUS were $1.04 \mathrm{~mm}(\mathrm{STD}=0.50 \mathrm{~mm})$. The system is in Phase-I clinical feasibility and safety trials, under Institutional Review Board approval.
\end{abstract}

\section{Introduction}

Transrectal ultrasound (TRUS) guided brachytherapy is an effective treatment for low-risk prostate cancer [1], but many implants continue to fail or cause adverse side effects. The procedure entails permanently implanting radioactive seeds into the prostate. It is commonly believed that pinpoint accuracy in executing a pre-operative implant plan should lead to good dosimetry. However, as two decades of practice have demonstrated, this is not achieved by all clinicians. Instead of enforcing a preoperative plan, intra-operative dosimetry and in-situ optimization have been receiving increasing attention [2]. This approach, however, demands precise localization of the implanted needles and seeds [3], which assumes exquisite spatial and temporal synchronization of the needle insertion and imaging tasks. The needles and seeds can be localized in TRUS, the dose field analyzed, and finally the remainder of the implant can be optimized. Needle positions are often rearranged to avoid overdosing and/or seeds added to fill cold spots. This, however, is a repetitive manual process that is prone to human operator errors and consumes valuable time in the operating room. Time delays may allow for increased edema that may change the anatomy and 
thereby negatively impact outcome. It is expected that intra-operative dosimetry can resolve these problems. This function, however, requires spatial and temporal synchronization of the actions of imaging, needle insertion, and needle/seed tracking. In current systems that attempt on-line dosimetry, these steps are performed sequentially by the physician who handles TRUS and needles together with the medical physicist who operates the treatment planning system (TPS). Motorizing the TRUS [4] and adapting the ultrasound phase/focus [5] have been shown to possess excellent potential in volume imaging and needle tracking. Hence the outstanding issue appears to be synchronizing needle action with ultrasound imaging. We first considered optical or electromagnetic (EM) tracking, but they turned out to be clinically impractical for a plethora of well known problems, such as lack of sight for optical trackers and field distortions for EM trackers; leaving us with some form of robotic assistance. Several medical robots have been proposed previously for prostate brachytherapy $[6,7,8,9,10]$ which strive to increase the accuracy of needle placement by transforming the workflow into a process we call "surgical CAD/CAM" [10]. Unfortunately, they add a great deal of complexity to the procedure and alter current hardware, calibration, and workflow standards. Our approach is different in that it adheres to the established standards of care, while also providing all practical benefits of robotic assistance. The novelty of our work lays in the adaptation and integration of existing robotic hardware in a simple and inherently safe manner.

\section{System Design}

The system consists of a central computer running the FDA-approved Interplant ${ }^{\circledR}$ TPS (CMS Inc., St. Louis, MO); a TRUS imager (B\&K Medical, 6.5MHz); an AccuSeed implant stand with digital probe positioner (also by CMS); and a small parallel needle guidance robot, as shown in Figure 1(left). We adapted a light weight parallel robot that rests on the mounting posts of the conventional template, as seen in Figure 1(right). The robot and the template are interchangeable during the procedure, as they are mounted in the same location and are calibrated to operate in the same coordinate frame. Thus, the unique feature of our system is retaining the existing clinical setup, hardware and workflow. In the case of a malfunction or even a slight suspicion of it, the physician can revert to the conventional template-based manual procedure without interruption. The robot is controlled by a standalone computer, thereby preserving the integrity of the FDA-approved Interplant system originally developed by Burdette Medical. The TRUS unit and the encoded stepper produce temporally and spatially tagged image streams for the TPS. In the experiments reported in this paper, an anthropomorphic mannequin was positioned supine, with a standard brachytherapy implant training phantom (CIRS Inc, Norfolk, VA) built into its perineum, as shown in the pictures of Figure 4.

The robot was originally developed for image-guided needle biopsy [11] and was customized by the manufacturer (PROFACTOR GmbH, Seibersdorf, Austria) to our specifications. The robot consists of two 2D Cartesian motion stages arranged in a parallel configuration (Figure 2). The $x y$ stage provides planar motion relative to the mounting posts, in the plane that corresponds to the face of the template. The 
workspace of $\pm 4 \mathrm{~cm}$ in each direction is sufficient to cover the prostate with a generous margin. The $\alpha \beta$ stage rides on the $x y$ stage, with a workspace of $\pm 2 \mathrm{~cm}$. The $x y$ and $\alpha \beta$ stages hold a pair of carbon fiber fingers that are manually locked into place during setup. A passive needle guide sleeve is attached between the fingers using free-moving ball joints. We decided against active needle driving. Instead, the robot functions as a fully encoded stable needle guide, through which the physician manually inserts the needle into the patient. The physician thus retains full control and

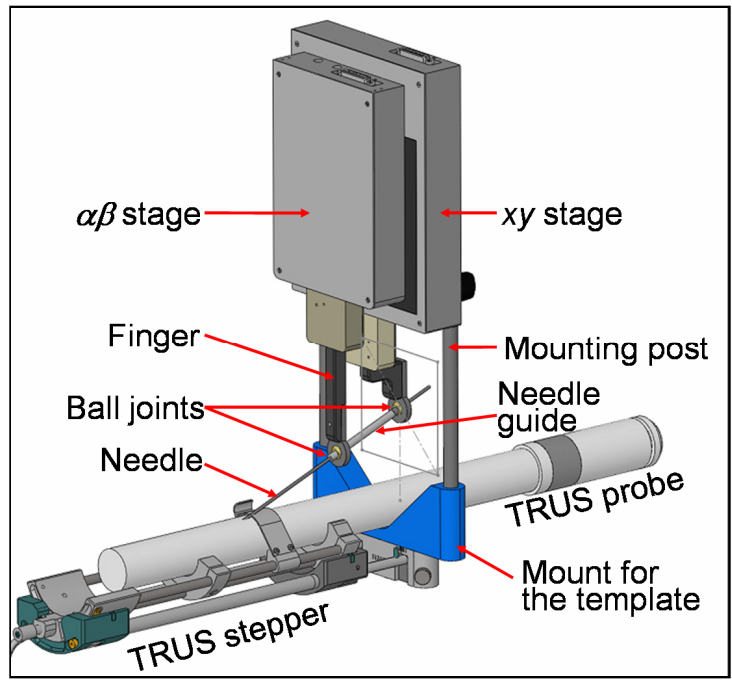

Fig. 1. CAD model of the parallel robot mounted over the TRUS probe on the mounting posts of the template natural haptic sensing, while the needle is being observed in live transverse and sagittal TRUS, ensuring exquisite control of the insertion depth relative to the target anatomy. If necessary, the insertion depth can also be encoded as in Seidl et al. [12], thus fully eliminating any practical need for active needle driving.

When the $\alpha \beta$ stage is in motion, the guide sleeve performs 2D rotation about the ball joint on the finger attached to the $x y$ stage. Needle angulation offers manifold advantages over the conventional template guidance where all needles were forced to

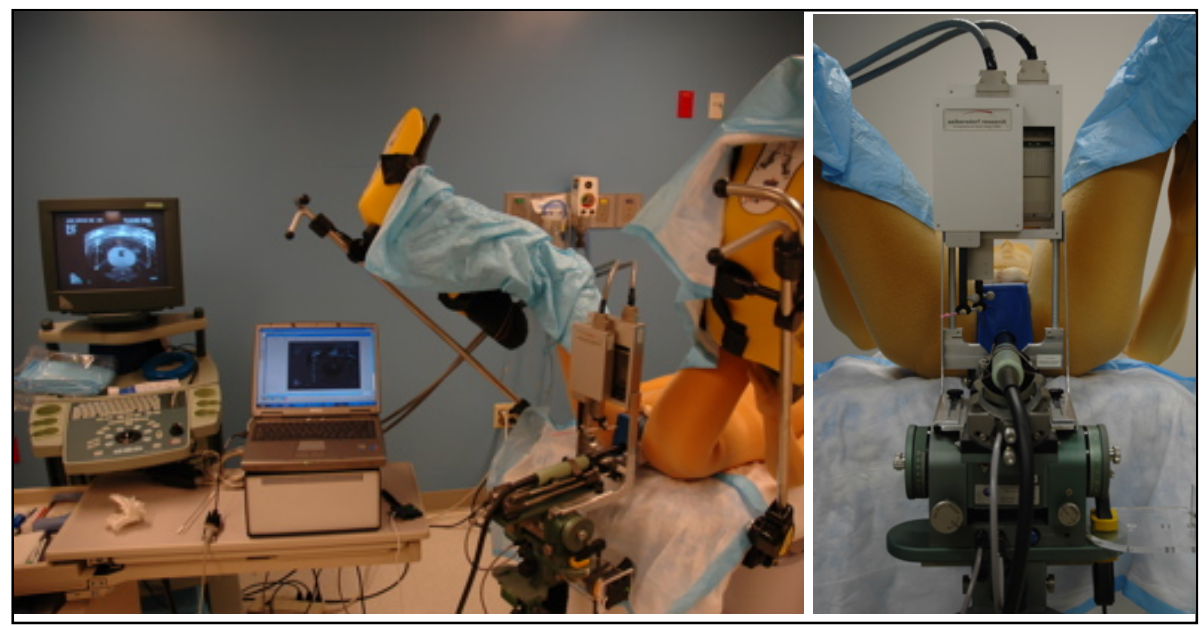

Fig. 2. System setup in the OR (left) and a closer view of the robot from the physician's perspective (right). Observe how the standard clinical hardware and setup are fully preserved. 
be parallel. Vertical angulation allows for avoidance of pubic arch interference, as seen in Figure 4(left). This happens when part of the prostate is hidden behind the pubic bone, which in contemporary practice can make patients with large prostates $(>55 \mathrm{cc}$ ) ineligible for brachytherapy. Additionally, biaxial angulation can account for anatomical asymmetries better than parallel needles, thereby yielding more conformal dose. The rotational workspace is a $\pm 20^{\circ}$ cone, sufficient to provide the required features. The length of the current needle guide sleeve is $70 \mathrm{~mm}$, providing steady support for the needle against buckling and slipping on the skin. While the guide sleeve takes up a longer length of the needle than the original template, it allows for shorter carbon fiber fingers that are stiffer and thus more accurate. If the needle guide length proves to be a clinical problem, we will redesign the fingers to reduce the length of the sleeve. The guide's diameter is slightly above $18 \mathrm{G}$, to accommodate standard brachytherapy needles without friction and play. (Note that the sleeve can be made to fit a needle of arbitrary size, such as a biopsy gun.) The disposable sleeve can be snapped in and out of the ball joints by hand. The robot is covered with a sterile plastic drape during the procedure; only the fingers and the guide sleeve are sterilized.

The robot weighs $1,300 \mathrm{~g}$. Its dimensions in home position are $140 \times 180 \times 65 \mathrm{~mm}$. Although it exerts some torque on the template posts, the load is bilaterally distributed over the stepper base with a supporting bracket, a precaution that prevents the robot from bending over the TRUS probe. The bracket can be seen Figure 4.

The robot control: The robot control architecture is shown in Figure 3. Low-level robot control is performed on a DMC-2143 controller board and AMP-20341 linear power amplifier (Galil Motion Control, Rocklin, CA, USA), which are connected via Ethernet to the laptop PC that runs the Robot GUI (Graphical User Interface) and the Interplant application software processes. Communication between these two processes is provided by a socket (UDP) connection. This required a minor modification to the Interplant software to add a "robot control" menu that invokes a small set of methods, defined in a dynamically loaded library (DLL), to initialize the robot, query its position, and move it to a new position. The DLL transmits these requests, via the socket connection, to the Robot GUI, which then invokes the appropriate methods in the Robot Class. Since the Robot GUI is in a separate process, it can also interact with the user directly; in particular, it updates the robot position/status display and accepts motion commands from the user.

In the current system, this is used to set the needle orientation because these two degrees of freedom are not controlled by the Interplant software. As noted in Figure 3, most of the custom software created for this project is written in $\mathrm{C}++$ or Python. There is also a small safety loop that compares the primary position sensors (incremental encoders) to the secondary

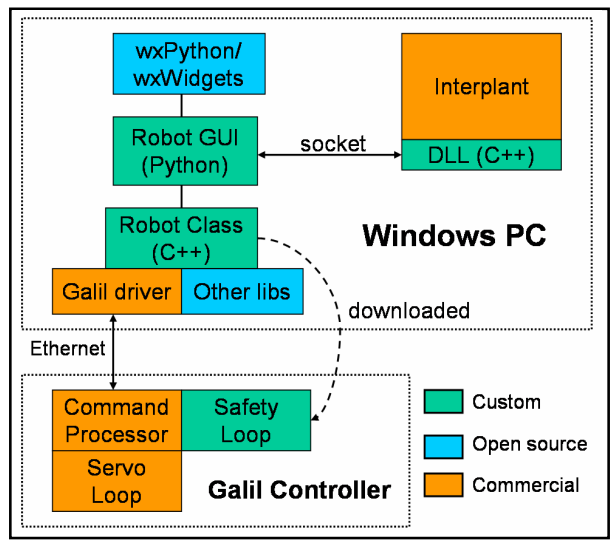

Fig. 3. The robot control architecture position sensors (incremental encoders). 
This is written in a Galil-specific interpreted language and is downloaded to the controller during initialization.

The calibration of the robot is identical to that of the conventional manual system and uses the same software kit and water tank. In essence, we move the needle tip inside the tank in a known trajectory by precise motion of the robot (serving as ground truth) and we also mark the needle positions in sagittal and transverse TRUS. Then by maximizing the similarity between the observations and ground truth, we obtain a transformation matrix between the TRUS and robot coordinate frames. Unlike any previous brachytherapy robot, ours does not require calibration before each procedure because the robot remains calibrated as long as its mounting remains calibrated to the TRUS.

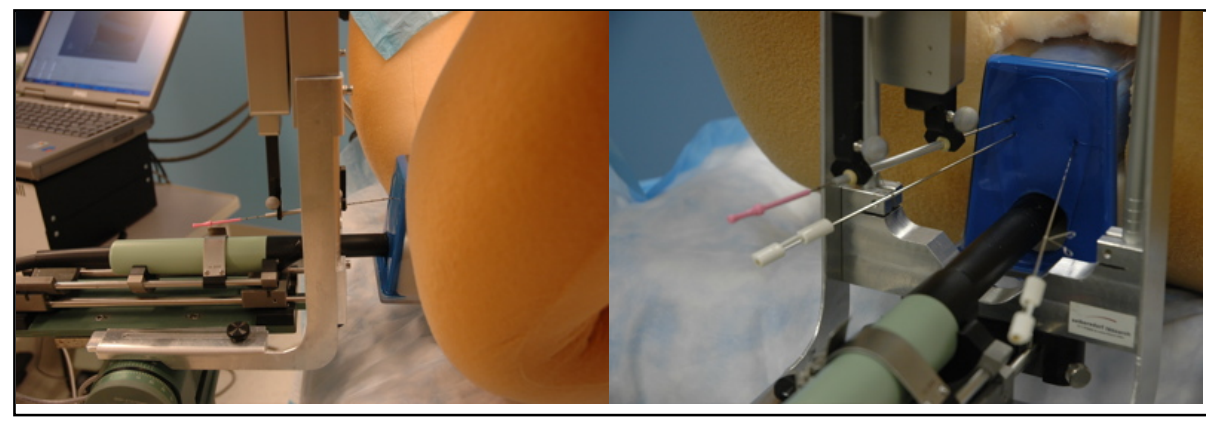

Fig. 4. Insertion of angulated needles. The needle is slanted upward to reach behind the pubic arch (left). Laterally slanted therapy needle in the presence of stabilizer needles (right).

The clinical workflow begins with segmenting the anatomy in TRUS and creating an implant plan. Bilateral stabilization needles may also be inserted. For each implant needle, the coordinates of the desired needle location are sent to the robot. The robot moves the needle guide onto the entry point over the perineum and orients it to the desired angle. The current Interplant dosimetry package does not support slanted needles, but the robot has this functionality. The physician inserts the preloaded needle or seed gun (such as Mick applicator) into the guide sleeve, and enters the needle into the desired depth while observing its progress in the live TRUS. The TPS has a near perfect estimate of the expected location of the needle in TRUS and a visual outline of the planned needle position is superimposed onto the spatially registered TRUS. The TPS processes the image to locate the needle and the operator may apply manual correction. The TPS then updates the dosimetry based upon the inserted needle position. The physician can make manual correction to the needle before approving the position and releasing the payload, or the physician may opt to pull out the needle without releasing the seeds. Only after correct needle position is confirmed, the physician will retract the needle and release the seeds. During the retraction of the needle, live TRUS images are acquired, wherein shadows of the seeds appear as they are released from the needle. The TPS processes the image to locate the seeds being dropped and the operator may also apply manual correction. Once the seeds are located, the computer promptly calculates a full dosimetry, using the seeds already implanted in their actual delivered locations, combined with the 
contribution of the remaining planned seeds. At this time, the physician can modify the remainder of the implant plan to compensate for cumulative deviations from the original plan. The cycle of execution is repeated with the next needle until satisfactory dosimetric coverage is achieved, which is the overall objective of the procedure.

\section{Experiments and Results}

We evaluated the prototype system in phantom trials. The robot fits in the neutral space over the perineum (Figure 1), without obstructing the swing space for a C-arm if one is present. The robot executed the designed ranges of motions. The Cartesian stage safely covered the axial dimensions of the prostate with generous margin. The function of needle angulation was also tested. Figure 4(left) depicts sufficient vertical angulation to point the needle behind the pubic arch while Figure 4(right) demonstrates vertical and lateral angulation. Note that unlike in any previous brachytherapy robot system, the implant needles can be inserted in the presence of bilateral stabilizer needles commonly used for reducing prostate motion during needle

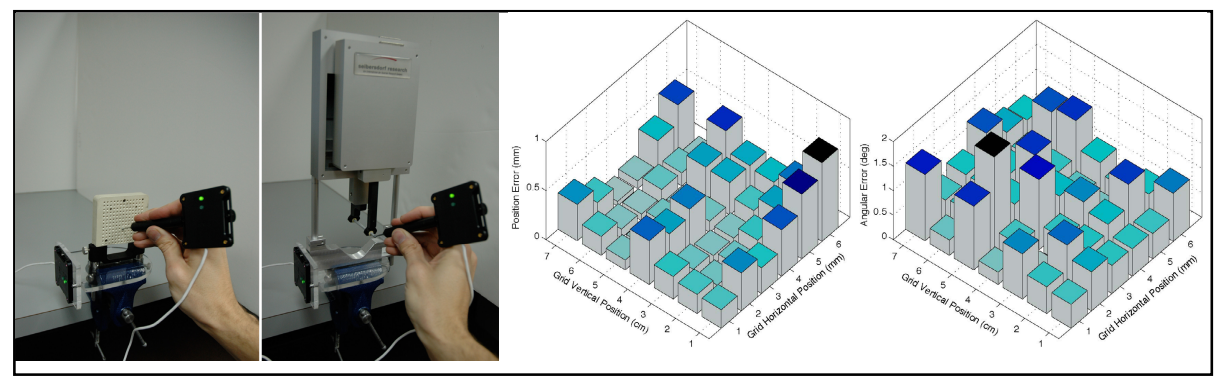

Fig. 5. Comparison of needle guidance between template and robot with Polaris tracker (left). Error bars for the translation (middle) and rotation (right) differences.

insertion [13]. In the case of collision, the distal finger gently deflects the stabilizer away, without causing tissue injury, while the physician is standing by to prevent the stabilization needle from being accidentally caught in the robot finger. (This issue will be studied further in a forthcoming Phase-I safety trial.)

We measured the accuracy of robotic needle positioning relative to the template. The robot, as mentioned earlier, is registered to the TRUS and the TPS commands address the robot in template coordinates. We performed 42 parallel positioning movements (7 rows of 6 columns, spaced $1 \mathrm{~cm}$ apart) in the $z$-axis with the robotic system and then manually with the template. We measured the positions of the corresponding template hole and the robotic needle guide before

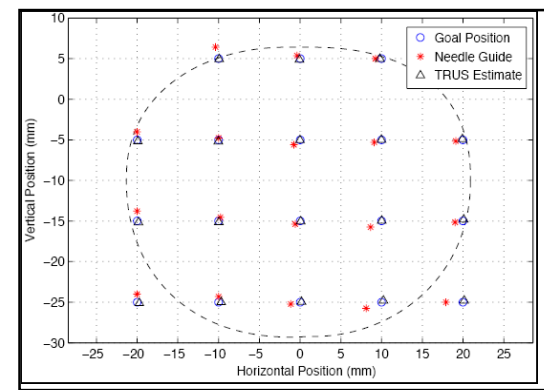

Fig. 6. Accuracy of robotically guided needle insertion relative to TRUS 
insertion with a calibrated ballpoint pointer (Traxtal Inc, Toronto, ON) tracked by a Polaris tracker (Northern Digital, Waterloo, ON), as seen in Figure 5(left). The error bars in Figure 5(middle) show a mean location error of $0.25 \mathrm{~mm}$ (STD $=0.17 \mathrm{~mm}$ ) which is less than the stated accuracy of the tracker. We also measured the accuracy of needle angulation relative to the z-axis. We performed 42 robotic positioning movements ( 7 rows of 6 columns, spaced $1 \mathrm{~cm}$ apart, in random angles between the extremes). We measured the angle of the guide sleeve by pivoting on both ends with the calibrated tracker pointer. The error bars shown in Figure 5(right) display a mean rotation error of $0.75^{\circ}\left(\mathrm{STD}=0.37^{\circ}\right)$, comparable with the accuracy of tracking.

We also measured the accuracy of robotic needle positioning followed by needle insertion into the phantom, relative to TRUS. We inserted 18 parallel needles along the $\mathrm{z}$ axis, marked their locations in TRUS and measured the location of the guide sleeve with the Polaris. As shown in Figure 6, all needles landed close to their goal, with a mean error of $1.04 \mathrm{~mm}(\mathrm{STD}=0.50 \mathrm{~mm})$. Locations near the prostate edge show somewhat larger errors attributed to slight needle deflection, which is still generously sufficient for brachytherapy. Placement accuracy of slanted needles suggested similar results, but we note that while slanted needles are currently not used in the dose planner, they are useful for adding individual seeds to patch up cold spots.

The apparatus allows for natural haptic feedback, but similarly to current template based practice, this feeling may be somewhat compromised by friction forces caused by needle bending and sliding forces. Lateral stabilization needles [13] provide some relief, as Podder et al. demonstrated in recent in-vivo needle force measurements [14].

In testing dynamic dosimetry, standard needles were inserted into a phantom. The moving needle was captured in live TRUS. A typical screen shot is shown in Figure 7, where the needle appears in the sagittal image as a white line. The expected seed positions relative to the needle tip are marked with squares. These squares were then used as initial search regions for localizing the seeds upon releasing them into the prostate. The resulting dose display was instantly updated so the clinician could follow the buildup of therapy dose, relative to the anatomy. Color-coded isodose lines seen around the needle are up dated as the seeds are captured.

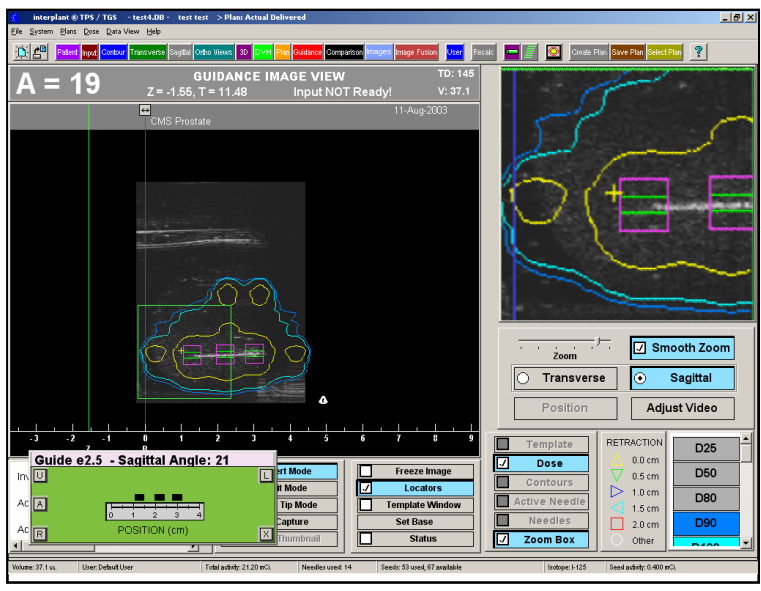

\section{Conclusion}

The robotic assistant provides

Fig. 7. Dynamic dosimetry screen from Interplant. The needle and seeds are captured in TRUS images as they are being inserted, while the resulting dose display is updated.

needle placement accuracy

equivalent to that of conventional templates while offering much greater flexibility, owing to its biaxial needle angulation and continuum Cartesian needle spacing. It is a 
digitally encoded system that allows for synchronized imaging and image-based needle/seed tracking, thereby opening the way for online dosimetry and implant optimization. These features were achieved without causing interference with established clinical hardware, workflow, or calibration standards. This is especially important as commercial potential and clinical viability in contemporary medicine are inseparable issues. Engineering development will continue with motorizing the TRUS base which already performs optical encoding of the stepper, making such a process relatively straightforward. Note that the system is functional without such motorization of the TRUS probe, though it requires some degree of manual adjustment during needle insertion and seed release, which from the dosimetric point of view is only an issue of convenience.

This work has been supported by DoD PC-050042, DoD PC050170, NIH 2R44 CA099374-02, and the NSF Engineering Research Center for Computer Integrated Surgical Systems and Technology under NSF EEC-9731748.

\section{References}

1. Zelefsky, M.J., Hollister, T., Raben, A., et al.: Five-year biochemical outcome and toxicity with transperineal CT-planned permanent I-125 prostate implantation for patients with localized prostate cancer. Int. J. Radiat. Oncol. Biol. Phys. 47, 1261-1266 (2000)

2. Zelefsky, M.J., Yamada, Y., Marion, C., et al.: Improved conformality and decreased toxicity with intraoperative computer-optimized transperineal ultrasound-guided prostate brachytherapy. Int. J. Radiat. Oncol. Biol. Phys. 55, 956-963 (2003)

3. Nag, S., Ciezki, J.P., Cormack, R., et al.: Intraoperative planning and evaluation of permanent prostate brachytherapy: Report of the American brachytherapy society. Int. J. Radiat. Oncol. Biol. Phys. 51, 1420-1430 (2001)

4. Wan, G., Wei, Z., Gardi, L., et al.: Brachytherapy needle deflection evaluation and correction. Med. Phys. 32, 902-909 (2005)

5. Okazawa, S., Ebrahimi, R., Chuang, J., et al.: Methods for segmenting curved needles and the needle tip in real-time ultrasound imaging. Medical Image Analysis, vol. 10(3), pp. 330-342. Elsevier Science Inc. New York (2006)

6. Fichtinger, G., Burdette, E.C., Tanacs, A., et al.: Robotically assisted prostate brachytherapy with transrectal ultrasound guidance-Phantom experiments. Brachytherapy 5(1), 14-26 (2006)

7. Wei, Z., Wan, G., Gardi, L., et al.: Robot-assisted 3D-TRUS guided prostate brachytherapy: System integration and validation. Med. Phys. 31, 539-548 (2004)

8. Yu, Y., Podder, T., Zhang, Y., et al.: Robot-Assisted Prostate Brachytherapy. In: Larsen, R., Nielsen, M., Sporring, J. (eds.) MICCAI 2006. LNCS, vol. 4190, pp. 41-49. Springer, Heidelberg (2006)

9. Phee, L., Yuen, J., Xiao, D., et al.: Ultrasound Guided Robotic Biopsy of the Prostate. International Journal on Humanoid Robotics 3(4), 463-483 (2006)

10. Fichtinger, G., DeWeese, T.L., Patriciu, A., et al.: Robotically Assisted Prostate Biopsy And Therapy With Intra-Operative CT Guidance. Journal of Academic Radiology 9(1), 60-74 (2002)

11. Kettenbach, J., Kronreif, G., Figl, M., et al.: Robot-assisted biopsy using ultrasound guidance: initial results from in vitro tests. Eur. Radiol. 15, 765-771 (2005) 
12. Seidl, K., Fichtinger, G., Kazanzides, P.: Optical Measurement of Needle Insertion Depth. In: IEEE International Conference on Biomedical Robotics, Pisa, Italy (February 15-19, 2006)

13. Taschereau, R., Pouliot, J., Roy, J., et al.: Seed misplacement and stabilizing needles in transperineal permanent prostate implants. Radiother Oncol. 55, 59-63 (2000)

14. Podder, T., Clark, D., Sherman, J., Fuller, D., Messing, E., Rubens, D., Strang, J., Brasacchio, R., Liao, L., Ng, W.S., Yu, Y.: Vivo motion and force measurement of surgical needle intervention during prostate brachytherapy. Med. Phys. 33(8), 2915-2922 (2006) 